\title{
Performance of Laser and Radar Ranging Devices in Adverse Environmental Conditions
}

\author{
Julian Ryde and Nick Hillier*
}

\begin{abstract}
A comparative evaluation of millimetre-wave radar and 2D scanning lasers in dust and rain conditions for sensor applications in field robotics is presented. A robust and reliable method for measuring the level of suspended dust (or other obscurant media) by determining the transmission coefficient is developed and used for quantitative assessment of sensor performance. The criteria of target acquisition reliability, precision and accuracy under varying environmental conditions are assessed via sensor operation in a controlled environment. This environment generated dust and rain of varying densities. Sensor performance is also assessed for the potential effect upon digital terrain mapping and haul truck localisation due to sensor-specific behaviours in these conditions (e.g. false targets, increased noise). Trials on a research electric face-shovel are conducted to test observed behaviours. It is concluded that laser scanners are suitable for environments with transmissions exceeding $92-93 \%$ per meter for targets closer than $25 \mathrm{~m}$. The radar remained relatively unaffected by the generated conditions of rain $(50-70 \mathrm{~mm} /$ hour $)$ and dust (10m visibility) however its accuracy $(0.1 \mathrm{~m}$ with a corner reflector and $0.3 \mathrm{~m}$ on a haul truck), free-space clutter and scan rate were insufficient for locating unmodified haul trucks for this application.
\end{abstract}

\section{Introduction and Background}

Scanning infra-red laser range finders and millimeter-wave radar have seen extensive application in automation and other mapping scenarios in a wide range of research and commercial environments. Typically, millimetre wave radar in the mining industry has been limited to slope stability monitoring [Reeves et al., 2000, Macfarlane and Robertson, 2004] (and systems by GroundProbe and Reutech), or imaging large underground cavities (stopes, orepasses [Brooker et al., 2005, Noon et al., 2002]), although there is a growing focus on its application to environmental mapping, volume estimation and machine component tracking (e.g., bucket localisation). In general, this mining focus has been spearheaded by work done at the Australian Centre for Field Robotics and CRCMining [Widzyk-Caperhart et al., 2006, Brooker et al., 2005, Brooker et al., 2007, Scheding et al., 2002].

\footnotetext{
* Julian Ryde and Nick Hillier are with the Autonomous Systems Laboratory, CSIRO ICT Centre, Brisbane, QLD, Australia. e-mail:\{julian.ryde, nick.hillier\}@csiro.au
} 
Scanning laser range finders operating in the (near) infra-red have found more wide-spread application in the mining environment, arguably due to the lower sensor costs and maturity of the technology. Although there has been minimal commercial up-take beyond simple seenot-see safety systems, research and prototype applications have ranged from moderate scale digital terrain mapping for automation purposes on draglines [Corke et al., 2000, Roberts et al., 2003] and other excavators [Singh, 1997, Stentz et al., 1999] through vehicle tracking for traffic control [Duff et al., 2006], machine component tracking [Duff, 2006, Hall and Keays, 1993], automatic dozer and grader blade control (systems by Trimble and others) and underground mine mapping [Huber and Vandapel, 2006, Shaffer et al., 1992, Baker et al., 2004, Nüchter et al., 2004].

Laser range finders and radar technologies are often used as complimentary sensing tools outside of the mining industry, particularly in vehicle automation [Miller et al., 2008, Yamauchi, 2007], however there have been minimal studies into the relative performance of these sensing technologies under adverse environmental conditions beyond their application to meteorological studies (of which there is a vast literature).

The Commonwealth Scientific and Industrial Research Organisation (CSIRO) in conjunction with CRCMining and the Australian Centre for Field Robotics (ACFR) conducted a series of controlled experiments to examine the performance of three scanning range devices, including a millimetre-wave radar, and two scanning infra-red laser range finders, when operating in various rain, mist and dust-cloud conditions. Subsequently, these sensors were installed on a research electric face shovel and further observations of sensor performance in a field deployment were made.

\subsection{Sensor Summary}

The sensors examined in this study are two scanning laser range-finders, the SICK LMS291S05 and the Riegl LMSQ120, and the 2D HSS - a 95GHz scanning millimeter-wave radar provided by ACFR. A brief summary of their key characterising specifications is presented in Table 1.

During the last two decades there have been significant improvements in the performance of time-of-flight laser range finding devices, in particular with regards to two areas: having poor sensing in adverse visibility conditions such as high suspended dust or water vapour loadings (fog, snow, rain); and being dazzled by direct viewing into the sun. Although, lasers are unable to range transparent objects like glass it is rare that this is a limiting factor in outdoor environments.

Lasers have much higher range precision and significantly tighter beam widths than radar sensors, allowing for the creation of maps with higher accuracies. Scanning lasers are also usually associated with relatively high scan rates and lower costs than radar. They are considered a mature technology with multiple suppliers and low lead times. Radar sensors are considered to be insensitive to suspended dust and water vapour loadings. The larger beam width and lower operating frequency of radar provides scope for measurement of multiple downrange targets along a single heading - even when visually obscured by intervening 


\begin{tabular}{|c|c|c|c|}
\hline & $\begin{array}{c}\text { Radar } \\
\text { 2D HSS }\end{array}$ & $\begin{array}{c}\text { SICK } \\
\text { LMS291-S05 } \\
\text { [SICK AG, 2000] }\end{array}$ & $\begin{array}{c}\text { Riegl } \\
\text { LMSQ120 } \\
{[\text { RIEGL Gmbh, 2005] }}\end{array}$ \\
\hline Min. range & $1 \mathrm{~m}^{\dagger}$ & $0 \mathrm{~m}$ & $2 \mathrm{~m}$ \\
\hline Max range & $70 \mathrm{~m}$ & $30-80 \mathrm{~m}$ & $75-150 \mathrm{~m}$ \\
\hline Range acc. & $>25 \mathrm{~mm}^{\ddagger}$ & $10 \mathrm{~mm}$ & $5 \mathrm{~mm}$ \\
\hline Beam width & $1.5^{\circ}$ & $0.7^{\circ}$ & $0.2^{\circ}$ \\
\hline Field of view & $360^{\circ}$ & $180^{\circ}$ & $80^{\circ}$ \\
\hline Min. angular res. & $1.2^{\circ}$ & $0.25^{\circ}$ & $0.04^{\circ}$ \\
\hline $\begin{array}{l}\text { Measurement } \\
\text { principle }\end{array}$ & $\begin{array}{c}\text { FMCW } \\
\text { with } \\
\text { CFAR peak } \\
\text { detection }\end{array}$ & $\begin{array}{c}\text { Single shot } \\
\text { time of flight } \\
\text { with fog and } \\
\text { pixel correction }\end{array}$ & $\begin{array}{c}\text { Single shot } \\
\text { time of flight } \\
\text { with multiple echo } \\
\text { discrimination }\end{array}$ \\
\hline Operating wavelength & $0.003 \mathrm{~m}$ & $905 \mathrm{~nm}$ & 'near IR' \\
\hline Max scan rate & $10 \mathrm{~Hz}$ & $75 \mathrm{~Hz}^{\star}$ & up to $100 \mathrm{~Hz}^{\star}$ \\
\hline
\end{tabular}

Table 1: Summary of commonly identifiable performance parameters between the tested sensors. (FMCW - Frequency Modulated Continuous Wave) (CFAR - Constant False Alarm Rate) ${ }^{\dagger}$ Radar minimum range is configurable. ${ }^{\ddagger}$ Depends on radar cross section (RCS). ${ }^{\star}$ Scan rate at best angular resolution is less.

objects. However, the radar as a sensing technology has a significantly wider beam-width and lower range precision than the laser sensors. The radar beam is transparent to some common materials (e.g. plastics typically yield low amplitude returns to radar).

The higher measurement uncertainties associated with radar often lead to more complex methods being pursued for map generation and data representation (for example volumetric evidence grids [Foessel, 2000]) by comparison to the simpler 2.5-D representations that are usually employed for digital map representation for laser scanners.

Both technologies have gained general acceptance for being safe, with the lasers presented here having Class I ratings (IEC 60825), and there being no known adverse health risks for exposure to the millimeter-wave radar beam of the instrument used here (IEEE C95.11991).

Active range sensors have to deal with uncertainties introduced when the illuminating beam spans a range discontinuity, for instance falling on both a near and far object. If the sensor is in first-echo mode then range to the nearest object in the beam's coverage is returned, however there are return modes which result in an effect known as mixed pixels (or phantom points) [Adams and Kerstens, 1996, Ye and Borenstein, 2002]. For safety motivated sensors such as the SICK this problem occurs more frequently due to the combination of a weighted first echo return and the larger beam divergence designed to ensure complete coverage. Range sensors returning the first-echo are more susceptible to interference from atmospheric obscurants and therefore it would be expected that the Riegl (which has configurable echo mode behaviour) would perform better than the SICK at higher dust and rain levels.

Range sensors in general can be susceptible to multi-path errors. These are errors that occur 
when the illuminating beam is specularly reflected from one surface and continues on and terminates on another elsewhere. This phenomenon gives an erroneous, more distant, range reading along the ray. This is especially dangerous for autonomous systems as it implies that the area is free of obstacles. Further background on the operation of the FMCW radar is contained within [Foessel, 2000].

To minimise uncertainty in the machine pose estimate, a commercial Applanix Inertially Aided Real-Time Kinematic GPS (IARTK) pose estimator was employed. This system is described and its performance assessed in [Arroyo-Suarez et al., 2005]. The IARTK estimator typically gave repeatability in position estimates to within $0.05 \mathrm{~m}$ and heading estimates to about 0.2 degrees at a $50 \mathrm{~Hz}$ update rate. Each pose estimate was time stamped, and network time was synchronised using the network time protocol (NTP) to be within 0.003s between computers. Sensor measurements used linear interpolation between pose updates. If there were more than one missing pose estimate then the corresponding range sensor measurements were discarded.

\subsection{Performance Impact from Obscure Intermediaries}

A good theoretic summary of the performance of millimeter-wave radar through clouds of coal dust and water droplets is presented by [Brooker et al., 2007], with a focus on conditions that can be expected in the mining environment. They also present summary findings for the dominant sensing technologies (millimeter-wave and micro-wave radar, laser, sonar) examining the attenuation of the sensor signals due to various dust loadings, and for the signal back-scatter encountered by millimeter-wave radar due to suspended dust and water droplets. For brevity, the analysis will not be repeated here. The conclusions reached from this theoretical work are that for the examined millimeter-wave radar (77 and $94 \mathrm{GHz}$ ), the effect of back-scatter is near negligible, and that the signal attenuation due to intervening media in the transmission space is only becomes significant at heavy suspended water loadings (visibility less than $4 \mathrm{~m}$ with large droplet sizes).

The authors were unable to find a similar study for laser range finding devices and there have been significant advances in the sensor technologies used for laser range finding in the last 25 years, not the least being the introduction of multiple-echo and last-pulse-return acquisition filtering.

\section{Method}

\subsection{Generating Adverse Conditions}

Experiments were undertaken in a confined environment (test chamber), see Section 2.2. Controlled rain, mist and dust conditions were replicated within this volume. A sprinkler system installed along the roof simulated rain and mist conditions whilst suspended dust conditions were created by a fan inducing airflow across a vibration table onto which dust was delivered. Dust(1-5kg) of varying particle size distributions and material was injected into the test chamber and the response of the sensors recorded during the period that the dust 
cleared (typically 10 to 15 minutes). It was found that the most dense dust-like conditions were created using media with the longest airborne suspension period (talcum powder). A high suspension time enables more material to be injected into to the volume before an equilibrium between the dust being injected and that settling out was reached, resulting in higher suspended densities.

Rain conditions were achieved by varying the flow rate through the overhead sprinkler system at constant pressure (via the use of a regulator). Low flow rates gave a droplet distribution biased towards larger particle sizes, high flow rates gave a distribution biased towards misty conditions with higher suspension ratios. The experimental procedure was unable to generate fog-like liquid suspensions. The generated conditions were colloidal with the generated rain and mist conditions being a mixture of a liquid aerosol with droplets not in suspension whilst the dust conditions were invariably a solid aerosol.

\subsection{Test Chamber Setup}

Fig. 1 and 2 present the test chamber and sensor arrangement. The test chamber was a rectangular room with a floor area of $30 \mathrm{~m}$ by $5 \mathrm{~m}$ and a height of $2 \mathrm{~m}$. The performance of both the radar and laser sensors was recorded for different rain, mist and dust conditions. A retro-reflective target (3M Scotchlite reflective sheeting 3800 series Class I) and normal diffuse surface were placed at a variety of distances from the sensors and the range readings to these were recorded for various conditions. It was found that accurate range determination was possible to the retro-reflective targets at higher dust and rain densities than for an obstacle with a surface exhibiting Lambertian reflectance.

Laser pointers were situated at several distances along the test volume to allow quantification of the dust concentration (see Section 2.3). The laser pointer spots were incident on a small white transparent screen.

Two cameras were used to observe the experimental conditions. The first (Allied Vision Technologies Marlin with a Fujinon 1:1.4/25mm lens) presented a view down the length of the test chamber, providing visibility information on contrast targets and qualitative confirmation of the obscurant media's even distribution along the length of the test volume via the relative scatter pattern of the laser pointer beams used for the transmission measurements. The second camera (Unibrain Fire-i web-cam) imaged the screen illuminated by the laser pointer spots to provide the intensity information used for the transmissibility evaluation.

Sample images from the camera viewing the test chamber are presented in Fig. 3 for three dust conditions. The first image is of the empty test chamber without any obscurant intermediary. This is assumed to be effectively clear space and taken as the baseline for the transmission calculations. The third image shows the test chamber under heavy dust loading (visibility $<8 \mathrm{~m}$ ). The middle image shows an intermediate condition. 


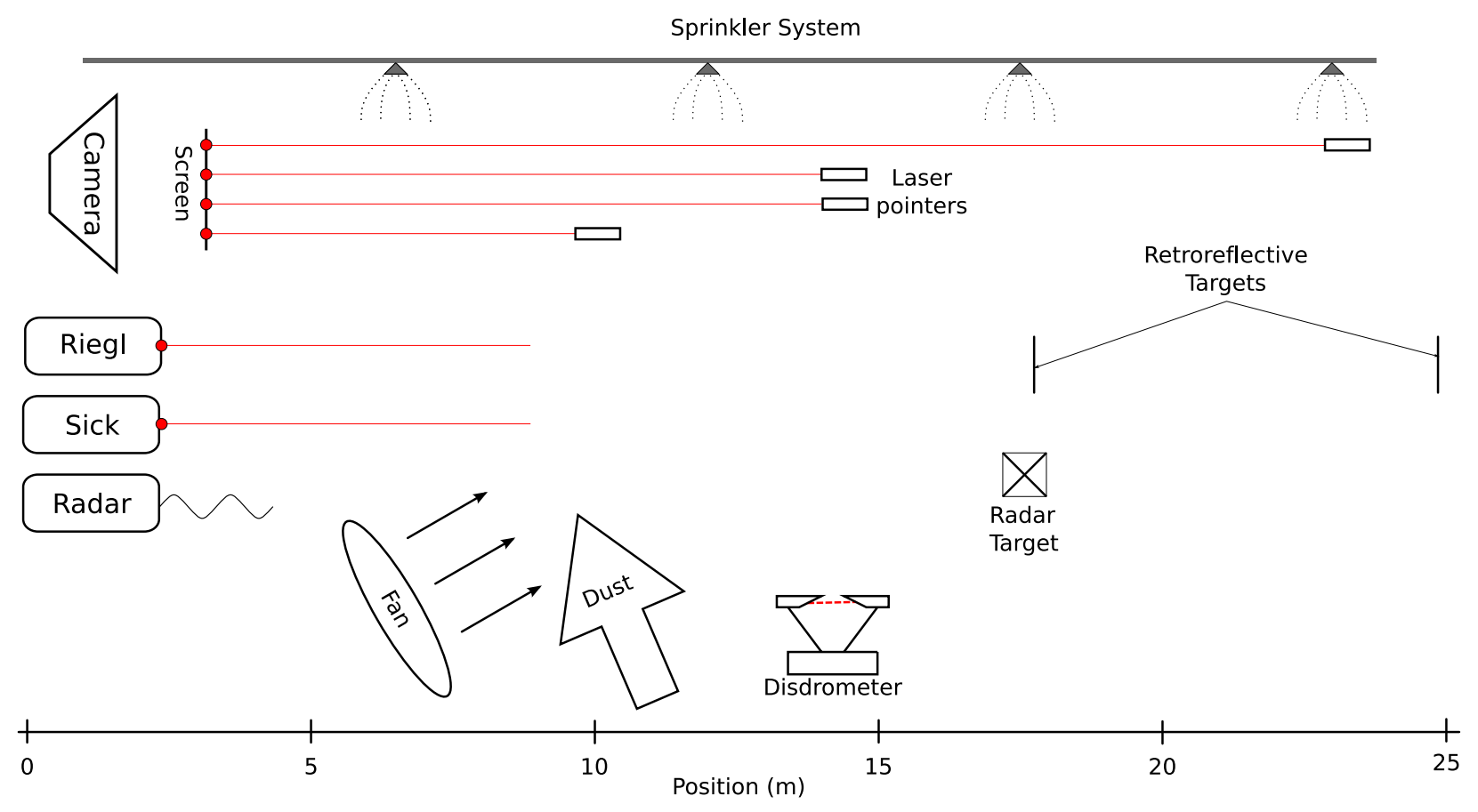

Figure 1: Diagrammatic representation of the test chamber setup.

\subsection{Measuring Obscurant Density}

Measurements from a disdrometer (Parsivel M300) were used to quantify the droplet size distribution and rainfall rate for the rain and mist experiments. The instrument's output was verified by comparison to rain gauge readings, flash images of the rain taken with a shallow depth of field, and comparison to the expected distribution for the sprinkler heads as specified by the test chamber design study [Martinez, 2007]. The imaging technique allows droplet size sampling by optical inspection. The shallow depth of field and selection of only those droplets in focus in the image ensures that the selected droplets are at the same distance from the camera.

To quantify the suspended dust loading, the transmissibility of the obscurant media was measured via the attenuation of the signal from $1 \mathrm{~mW}$ red laser pointers placed at varying distances $(\approx 6,17,25 \mathrm{~m})$ along the test chamber. A similar method has been used successfully in the past by [Pinnick et al., 1983] which compared the transmission of visible and infra-red signals at specific frequencies through explosion generated dust, as well as using a plethora of independent measurement techniques (Hi-Vol sampling and subsequent lab analysis, Knollenburg counters of various sensitivities and measurement principles) to characterise the dust.

The Beer-Lambert Law presents an empirical relationship between the absorption of visible light at a given wavelength through a material, to the physical properties of the material, most notably for this study: the material's density and absorption properties. This provides a convenient means for characterising obscurant media, in that measurements of the intermediary's transmissibility can be directly related to the performance of laser range finding 


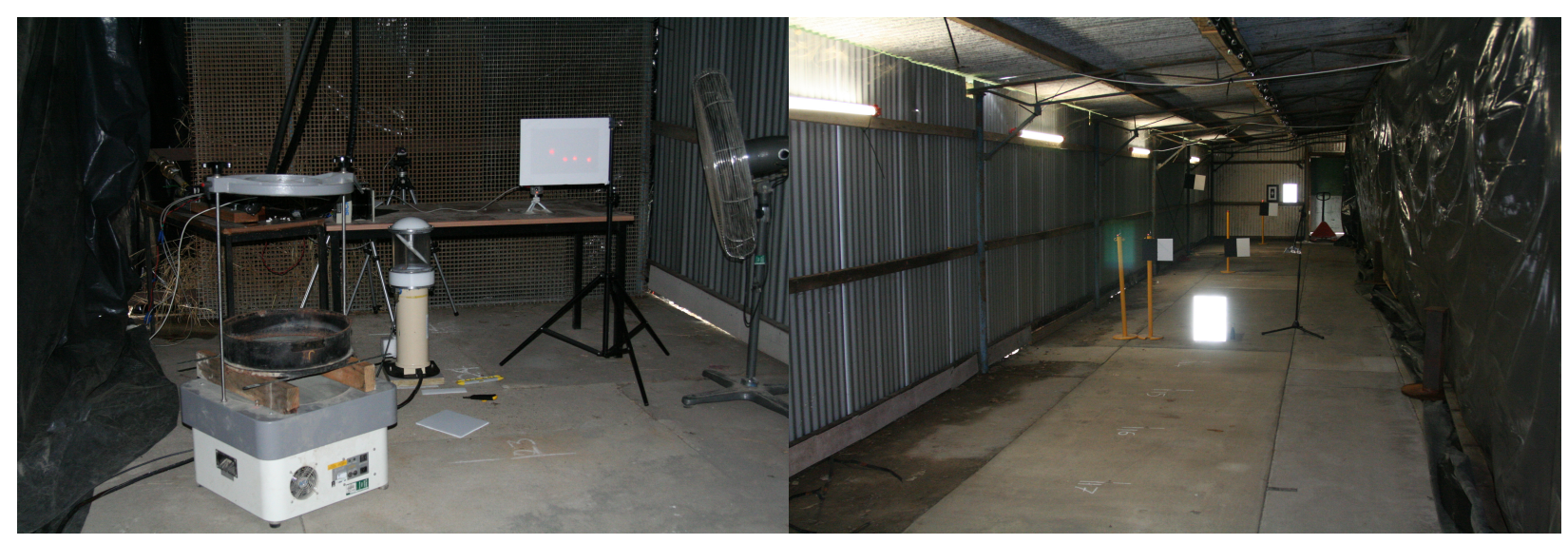

Figure 2: The experimental setup. Sensor placement (left), showing the shaker table (left of image), imaging cameras, laser sensor, radar and screen for laser pointer spots. The volume towards the targets (right), showing contrast targets, radar reflectors and retro-reflective targets for the laser sensors.
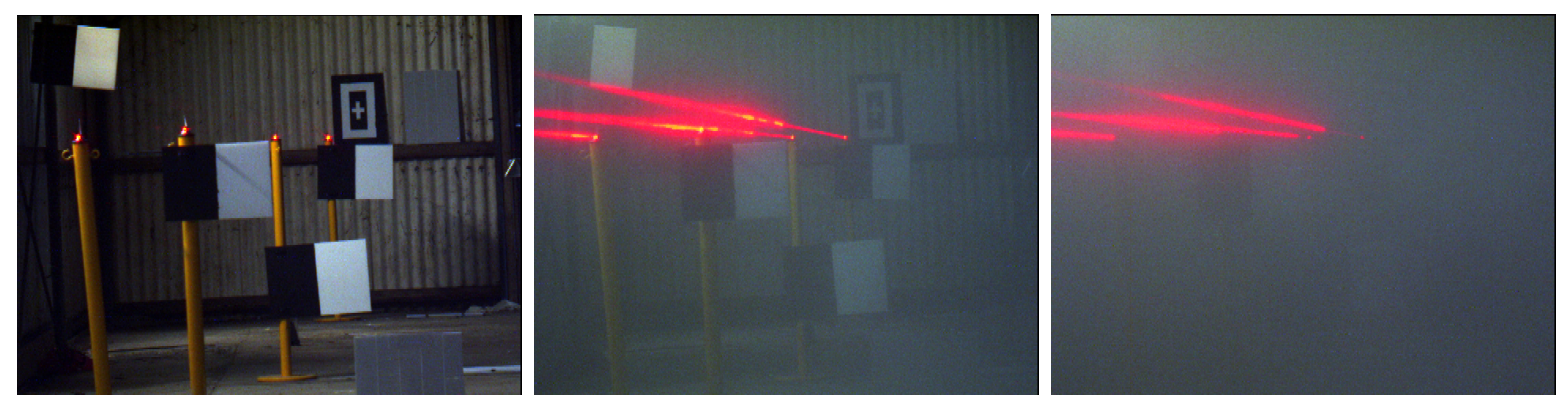

Figure 3: Typical images captured during dust experiments. The first image (left) is of the empty test chamber without any obscurant intermediary. The centre image shows an intermediary condition during dust injection. The third image (right) shows the test chamber under heavy dust loading (visibility $<8 \mathrm{~m}$ ) 
devices without the need to formally characterise the obscurant's composition and density.

For the purposes of this study, the transmissibility, $T$, of the media was measured using the ratio of the measured intensity of the laser pointer before $I_{0}$ and after $I_{1}$ passing through the obscurant intermediary (1).

$$
T=\frac{I_{1}}{I_{0}}
$$

This can be related to the intermediary material's properties through (2):

$$
A=-\ln (T)=\sigma l N
$$

where $A$ is the characterising absorbance of the media, $\sigma$ is the obscurant's absorption cross section, $l$ is the length between the incident and measured intensities and $N$ is number of absorbers.

Visibility can be roughly related to the mass of dust per volume, which is the typical measurement unit for quantifying dust density in occupational health and safety fields, through [Gillett, 1979, Brooker et al., 2007]:

$$
M=\frac{C}{V^{\gamma}}
$$

where $M$ is the mass of suspended particulate $\left(\mathrm{g} / \mathrm{m}^{3}\right), C$ and $\gamma$ are constants of proportionality dependant on the suspended materials and atmospheric composition and $V$ is the visibility (m). [Brooker et al., 2007] gives typical values for $C$ and $\gamma$ as 37.3 and 1.07 respectively.

Unlike measurement of transmissibility (1) the transmission coefficient is independent of path length and provides a better descriptor for the concentration of suspended dust and the characteristic absorption of the dust particles. The transmission coefficient, $T_{c}$ is calculated from a combination of (1) and (2) as per (4).

$$
T_{c}=\left(\frac{I_{1}}{I_{0}}\right)^{1 / l}=e^{-\sigma N}
$$

The imaged laser pointer spots were analysed and used to compute the dust transmissibility. For each spot, a rectangular region of interest (ROI) containing the full extent of the spot was defined. For each image during the experimental period, the red components were summed over the ROIs and the baseline red component from the background (from a region of the screen with no incident spot) subtracted. This method provided normalisation of the spot intensities with respect to each other and the background and allowed calculation of the total incident energy despite the variety of spot sizes due to the varied distances between the screen and the laser pointers. The sums of the red components for the regions of interest 


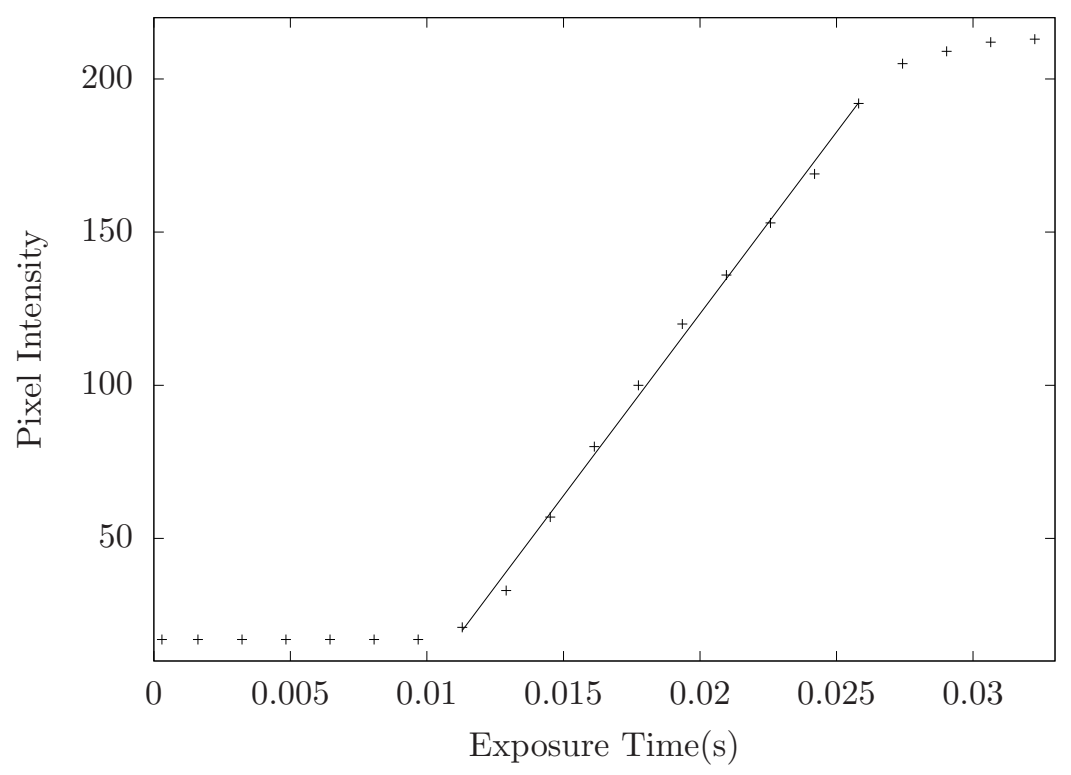

Figure 4: Camera response curve illustrating the region of linearity.

was normalised such that the transmission coefficient was one $(100 \%$ of the transmitted light is incident at the receiver) when there was no obscuring media other than air.

Calculating the relative incident light energies for the transmission coefficient requires knowledge of the response curve of the camera imaging the laser pointer spots. This response curve relates the incident light energy to the pixel intensity recorded by the camera sensor. The curve was obtained by imaging a white target under constant illumination with varying exposures. If all other settings are held constant, the total incident light energy is directly proportional to the exposure time. In this manner, Fig. 4 was generated and shows the response function for the camera imaging the laser spots. There is significant non-linearity in the imaging camera's response for pixel intensities outside of the 30 to 200 range and thus only intensities measured within this bound were compared for the calculation of the relative incident light energy.

For the validity of this method, it is vital to use lossless compression of the images to prevent blurring and colour bleed artefacts that may influence the results. The laser pointer spot images also need to be free of saturated pixels, to prevent incident energy being unaccounted. Saturation limits can be tuned by varying the imaging camera's exposure settings.

It was found that during initial dust injection into the test chamber, and for a short period after, the density of dust along the length of the test volume was non-uniform as confirmed by visual inspection of the scatter lines from the laser pointers which exhibited variation along their length as a function of the density at that distance. This is illustrated in the second image of Fig. 3 where the width and intensity of the laser's scatter line varies with the dust density along the length of the test chamber. After approximately 20 seconds the dust establishes a colloidal suspension with near constant scatter apparent along the image of the laser line (see the third image of Fig. 3). The resultant solid aerosol is relatively stable, 


\begin{tabular}{|l||c|c|c|}
\hline & Radar & SICK & Riegl \\
& 2D HSS & LMS291-S05 & LMSQ120 \\
\hline \hline Dist. to reflective target 1 & & & \\
by instrument & $16.154 \mathrm{~m}$ & $17.040 \mathrm{~m}$ & $17.603 \mathrm{~m}$ \\
by tape-measure & $16.15 \mathrm{~m}$ & $17.05 \mathrm{~m}$ & $17.60 \mathrm{~m}$ \\
Mean RMS error & $0.086 \mathrm{~m}$ & $0.006 \mathrm{~m}$ & $0.008 \mathrm{~m}$ \\
Signal strength (intensity) & $83.4 \mathrm{~dB}$ & 1 & 214 \\
\hline Dist. to reflective target 2 & & & \\
by instrument & $18.359 \mathrm{~m}$ & $24.070 \mathrm{~m}$ & $24.591 \mathrm{~m}$ \\
by tape-measure & $18.35 \mathrm{~m}$ & $24.07 \mathrm{~m}$ & $24.60 \mathrm{~m}$ \\
Mean RMS error & $0.066 \mathrm{~m}$ & $0.007 \mathrm{~m}$ & $0.010 \mathrm{~m}$ \\
Signal strength (intensity) & $95.17 \mathrm{~dB}$ & 1 & 204 \\
\hline Other notable effects & Multi-path. & Low & None \\
& Saturation. & Signal & \\
\hline
\end{tabular}

Table 2: Target acquisition performance, clear conditions. Intensity varies over 0 to 7 for the SICK and 0 to 255 for the Riegl.

existing for a number of minutes after the dust injection process is halted.

\section{Experimental Results}

\subsection{Clear Conditions}

Baseline results for the clear conditions were recorded to characterise the sensor performance and are presented in Table 2. These results are comparable to the manufacturer specified sensor characteristics. Distance was measured to the instrument (to the nearest 10mm) and compared to the instrument's measurement of the distance (to the nearest $1 \mathrm{~mm}$ ). The mean RMS error from the instrument's measurement mean and the return signal intensity is also presented.

Multipath and saturation effects were visible in the radar data for some scan points away from the target reflectors. These are wholly due to the geometry and composition of the metal walls and roof of the test chamber enclosure (and would not be apparent in a typical deployed environment). Multipath errors were also visible in the Riegl data where the beam reflected off the smooth surface of the roof or floor. This error was intermittent and localised to specific areas of the test chamber. Changing the device to first-echo mode eliminated these effects. All further testing was conducted in last-echo mode.

These erroneous effects were filtered out of the analysed data sets to create a base-line for the clear conditions. 


\subsection{Rain and Mist Experiments}

A variety of rain flow rates were used to generate a series of droplet diameter versus frequency distributions and the resultant sensor outputs for each condition compared. The results of this testing are presented in this section.

Through the range of test conditions, negligible effect was observed to the radar sensor output. Despite a slight drop in peak signal strength (in line with the theory presented in [Brooker et al., 2007]), no change to return accuracy and precision or target discernability were noted. There were no observable changes to the existing multi-path and saturation effects from the clear condition testing.

Both laser sensors were generally robust to the low flow rate conditions, but presented degrading performance as flow rates increased and heavy mist-like conditions were generated. It was found that the rain rate had only a minor performance impact on the accuracy and precision of the ranging measurement of the sensors, however did significantly alter the probability of detecting the target (or returning a false or absent range measurement) and so we focus our analysis on this point.

Fig. 5 presents the drop size and frequency distribution for the threshold conditions (approximately $50 \%$ valid returns) for the SICK laser. These conditions represent those in which it can be argued that the SICK laser sensor data can still be considered usable. This occurred at rainfall rates of approximately $50-70 \mathrm{~mm} /$ hour. Although it may be expected that the presented droplet diameter distribution would be a monotonically decreasing curve, the test setup was unable to produce very small drop sizes or fog-like conditions, and the disdrometer was limited by a lower measurement bound for droplet diameters of $0.25 \mathrm{~mm}$. Table 3 presents a summary of the average sensor performance over a 5 minute test in these conditions. Distance was measured to the instrument (to the nearest $10 \mathrm{~mm}$ ) and compared to the instrument's measurement of the distance (to the nearest $1 \mathrm{~mm}$ ). The mean RMS error from the instrument's measurement mean, the return signal intensity and the probability of target acquisition over the 5 minute window is also presented.

It was found that the SICK sensor was more robust to larger drop sizes than the Riegl, whilst the Riegl showed better performance than the SICK in conditions with dense, small drop sizes (misty conditions). There was significant variation in the probability of target acquisition over the test period when using a 1000 sample moving probability window for the SICK. The range measurements for false target acquisitions also showed a high variation. This suggests that the SICK sensor's mode of failure was detection of rain drops instead of the target, manifesting itself in either the range return of the raindrop itself or a mixed-pixel effect between the raindrop and the target. This is consistent with the larger beam width of the SICK, whereas the Riegl showed near constant failure probabilities during the tests with failures tending to be absent measurements or very low range false target acquisitions. This behaviour is consistent with the tight beam width of the Riegl being intercepted by the rain drops. The Riegl's superior performance in the misty conditions was limited to the last-echo return mode and exemplifies the performance gains available through such filtering methods. 


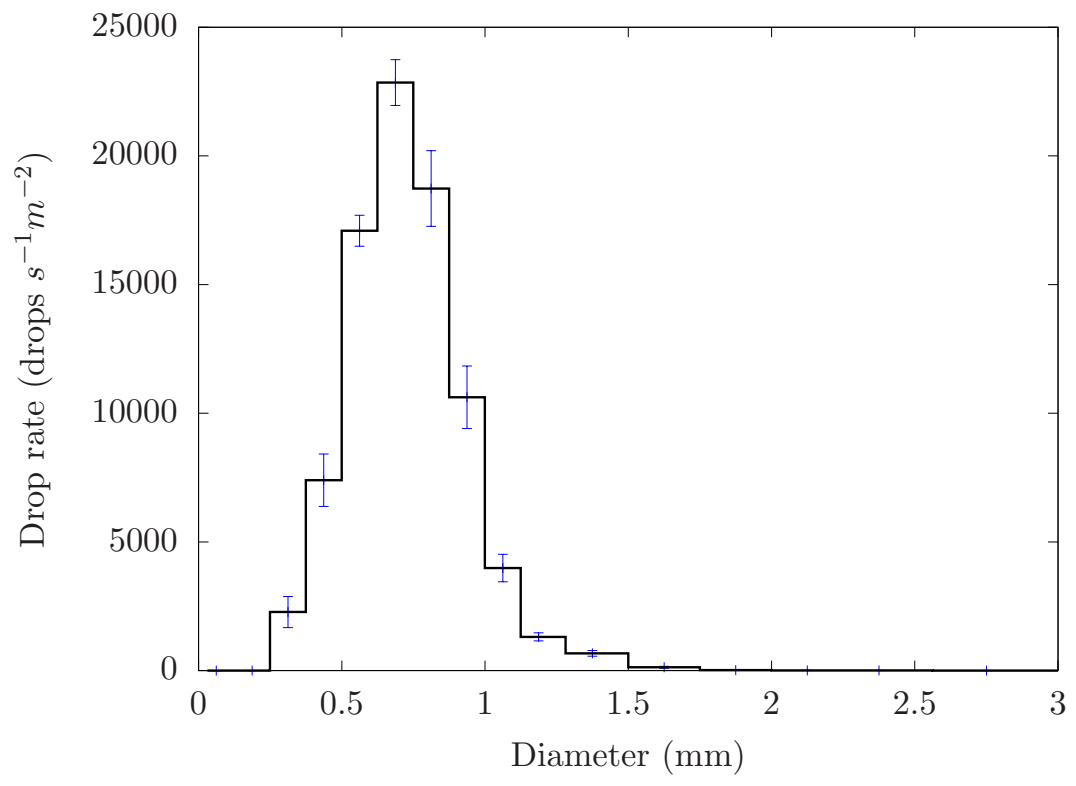

Figure 5: Droplet diameter distribution for the threshold (approximately $50 \%$ failure rate) laser returns. Note that the curve goes to zero at low droplet diameters due to the lower measurement bound of the instrument and the limitations of the method used to generate the rain conditions to produce very fine drop sizes.

\begin{tabular}{|l||c|c|c|}
\hline & $\begin{array}{c}\text { Radar 2D } \\
\text { HSS }\end{array}$ & $\begin{array}{c}\text { SICK } \\
\text { LMS291-S05 }\end{array}$ & $\begin{array}{c}\text { Riegl } \\
\text { LMSQ120 }\end{array}$ \\
\hline \hline Dist. to reflective target 1 & & & \\
by instrument & $14.103 \mathrm{~m}$ & $17.110 \mathrm{~m}$ & $17.610 \mathrm{~m}$ \\
by tape-measure & $14.10 \mathrm{~m}$ & $17.10 \mathrm{~m}$ & $17.60 \mathrm{~m}$ \\
Mean RMS error & $0.04 \mathrm{~m}$ & $0.01 \mathrm{~m}$ & $0.022 \mathrm{~m}$ \\
Signal strength (intensity) & $83.38 \mathrm{~dB}$ & 0 & $60-190$ \\
Probability of target & $100 \%$ & $81 \%$ & $70 \%$ (last echo), \\
acquisition & & & $40 \%\left(1^{\text {st }}\right.$ echo) \\
\hline Dist. to reflective target 2 & & & $24.600 \mathrm{~m}$ \\
by instrument & $14.777 \mathrm{~m}$ & $24.170 \mathrm{~m}$ & $24.60 \mathrm{~m}$ \\
by tape-measure & $\mathrm{NA}$ & $24.17 \mathrm{~m}$ & $0.038 \mathrm{~m}$ \\
Mean RMS error & $0.098 \mathrm{~m}$ & $0.007 \mathrm{~m}$ & $60-160$ \\
Signal strength (intensity) & $82.13 \mathrm{~dB}$ & 0 & $65 \%$ (last echo), \\
Probability of target & $100 \%$ & $49 \%$ & $44 \%$ (1 ${ }^{\text {st }}$ echo) \\
acquisition & & & multi-path, \\
Other notable effects & none & multi-path & \\
& & & \\
\hline
\end{tabular}

Table 3: Target acquisition performance, rain and mist threshold conditions (approximately $50 \%$ valid returns for the $25 \mathrm{~m}$ target, as presented in Fig. 5) for a five minute test. Intensity varies over 0 to 7 for the SICK and 0 to 255 for the Riegl. 
Wet surface conditions and puddles form a reflective surface at low angles of incidence and refractive surface at higher angles for the laser beam. This introduces multi-path effects which were observable in the acquired laser data as reflections of the targets about the ground plane in both laser sensors. The Riegl also displayed a false target ('ghost' points) floating above the retro-reflective target at $17 \mathrm{~m}$ (a reflection about the centre of the sensor's field of view) in the clear conditions between rain and mist tests when the surrounds were wet. This false target disappeared if the retro-reflective target at this distance were replaced with a target of lower reflectivity, more Lambertian reflectance, or when the target, ground and walls of the test chamber had dried. It can be speculated that this false target could be due to reflections internal to the sensor's mechanism under high intensity return conditions or an effect similar to lens flare as observed on cameras.

\subsection{Dust Experiments}

A variety of tests were conducted to determine the method to produce the densest dust cloud (see Section 2.1) and subsequently, a series of sensor performance experiments conducted, the results of which are detailed in this section.

Through the range of test conditions, negligible effect was observed to the radar sensor output. No change to signal strength, return accuracy and precision or target discernibility were noted. There were no observable changes to the existing multipath and saturation effects observed for the radar from the clear condition testing.

Over the course of the dust clearing, it was found that $T_{c}$ rises from $91 \%$ to $96 \%$. As this is normalised for a path length of $1 \mathrm{~m}$, this change appears insubstantial, however over the $50 \mathrm{~m}$ path length for which the lasers pass for a target at a range of $25 \mathrm{~m}, T_{c}^{50}$ corresponds to values of transmissibility in the range of $1-10 \%$.

It was found, for the laser sensors, that target acquisition failure was abrupt - probability of target acquisition varied from $100 \%$ to complete failure within a very small change in the transmissibility measurement (see Fig. 6). The threshold transmission coefficients are 0.921 for the LMS and 0.926 for Riegl for which the corresponding transmissibilities over a $50 \mathrm{~m}$ path length are 0.0163 and 0.0213 respectively. These results emphasise the effectiveness of the ranging sensors when the return energy is only 1-2\% of the energy emitted by the device. This analysis excludes energy losses from absorption at the target which will further reduce the returned energy. At the levels of transmission for which the lasers failed for the $25 \mathrm{~m}$ target, visibility was poor: typically of the order of $10 \mathrm{~m}$.

Fig. 7 presents the 10 second RMS error from the measurement mean for each of the laser sensors as a function of the transmission coefficient. As expected and similar to the curve found in [Adams and Kerstens, 1996], the error in the measurement increases for both sensors as the amount of dust increases (and the transmission decreases) before the abrupt loss of target. This is further illustrated in Fig. 8 which presents histograms of the range returns for various transmission coefficients. Additionally, these distributions show that the SICK LMS shows a systematic range increase as the dust level increases that is separate to those due to range and incidence angle presented by [Diosi and Kleeman, 2003]. The histograms in Fig. 8 


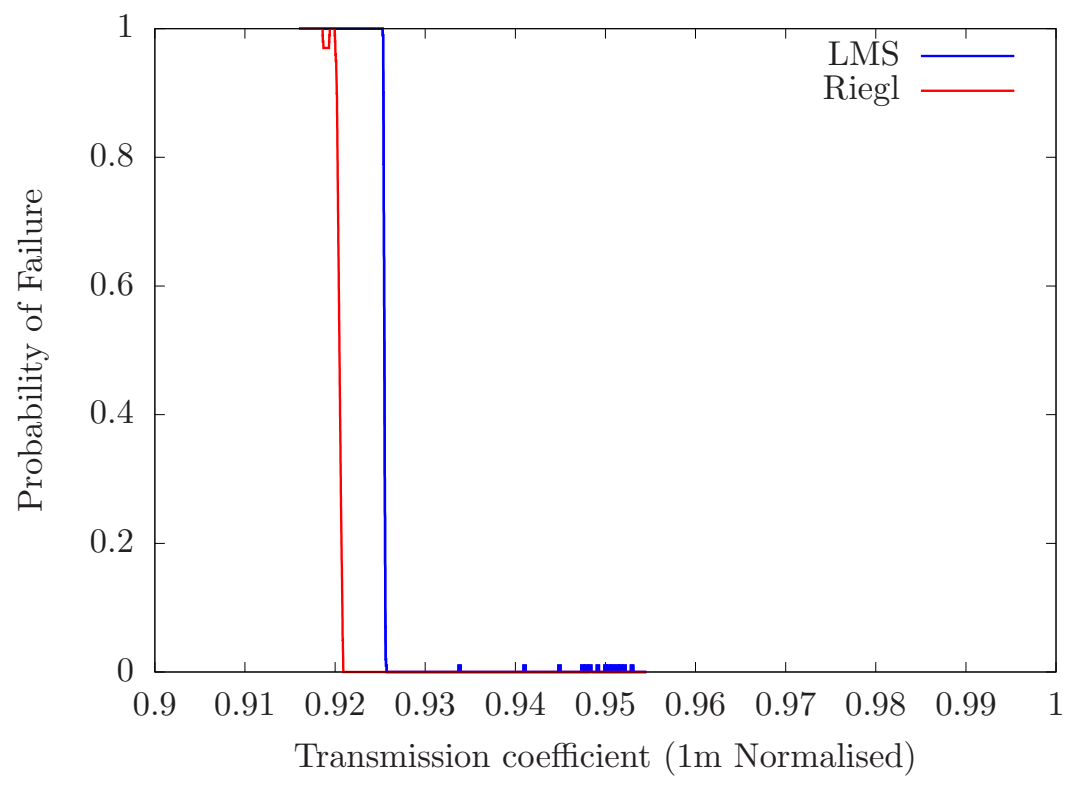

Figure 6: Probability of failure for both the SICK and Riegl against transmission for the dust experiments. The target is $25 \mathrm{~m}$ from the laser scanner. There are increasing range reading failures as the transmission falls.

provide an effective means of characterising the laser sensor performance as a function of obscurant density for the purpose of building a sensor model. The Reigl is better behaved: as the dust levels increase, no systematic bias is observed, but an increasing spread which is correctly centred. Clear-space geometric and systematic sensor behaviours for the SICK are well documented in [Ye and Borenstein, 2002] and more generally for laser range finders in [Adams and Kerstens, 1996, Arras, 2003] and the methods presented appear suitable for defining the Riegl's characteristics. The normal fits for the distributions in Fig. 8 indicate that the range distribution may be adequately described by a Gaussian model within the error bars of these experiments. No other sensor performance degradation effects (such as multipath) were observed during the dust tests, although effort was made to keep sensor lenses and targets clean.

\section{Field Experiments on an Electric Rope Shovel}

Four sensors were installed onto a P\&H 2100 BLE electric face shovel (Fig. 9) for the purpose of generating digital terrain maps and performing volume estimation tasks. These maps are passed to an automation system to allow path planning and collision avoidance. Two SICK LMS291-S05 were mounted either side of the crowd-arm pivot, a Riegl LMSQ-120 mounted adjacent to the right hand side SICK and an ACFR radar as presented in the preceding sections was mounted on the left hand side of the machine house. The laser scanners rely on shovel motion for 3D data collection whilst the radar was modified with a pan axis to allow 3D data collection without swinging the shovel. Machine pose information was provided to the sensors via the IARTK system (discussed in Sec. 1.1) installed on the shovel which 


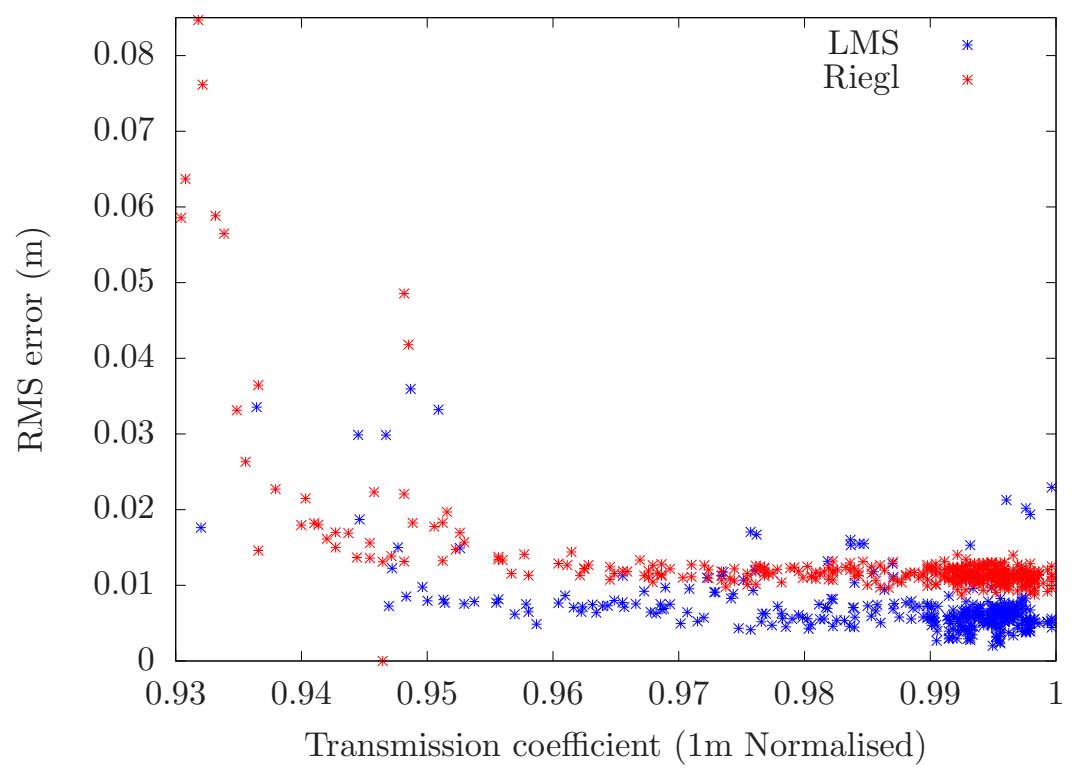

Figure 7: RMS error from a ten second sample mean for both the SICK and Riegl against transmission for the dust experiments. The target is $25 \mathrm{~m}$ from the laser scanner. The RMS error increases as there is an increase in obscuring media until failure as given by Fig. 6

allowed measurement conversion to a common reference frame.

No sensor problems due to vibration or shocks were evident on this installation. In part, this is likely due to the relatively smooth operation of this type of excavation machinery, which has very slow dynamics due to the significant vehicle mass. The onboard pose estimator incorporates an IMU and provides pose estimates at $50 \mathrm{~Hz}$ (and internally at up to $200 \mathrm{~Hz}$ ), whereas the dominant machine dynamics are in the order of $<5 \mathrm{~Hz}$. This oversampling minimises map registration errors and possible ghosts, distortions and other artifacts in the scan images due to vibration.

The ghosting effects observed during the controlled indoor tests were also apparent in the field data on items of high reflectivity (e.g. vehicle number plates and some signs - see Fig. 10). The SICKs provide a larger field of view than the Riegl (180 versus 80 degrees) allowing proprioception of the shovel including the crawler tracks and rear-mounted cablereeler. The wider field of view also allows sighting of items at higher altitude, including the tops of surrounding trees.

\subsection{Field results and analysis}

Data from the radar was processed in a first-point-return mode to ease interpretation from possible multiple echoes along the same heading. The radar data required thresholding to remove free-space clutter. Fig. 11 presents the distribution of returns against intensity for the radar that was used to select an initial guess for the free-space clutter cut-off value of $75 \mathrm{~dB}$ which was further refined (to $78 \mathrm{~dB}$ ) by manually examining the data. In general, the 


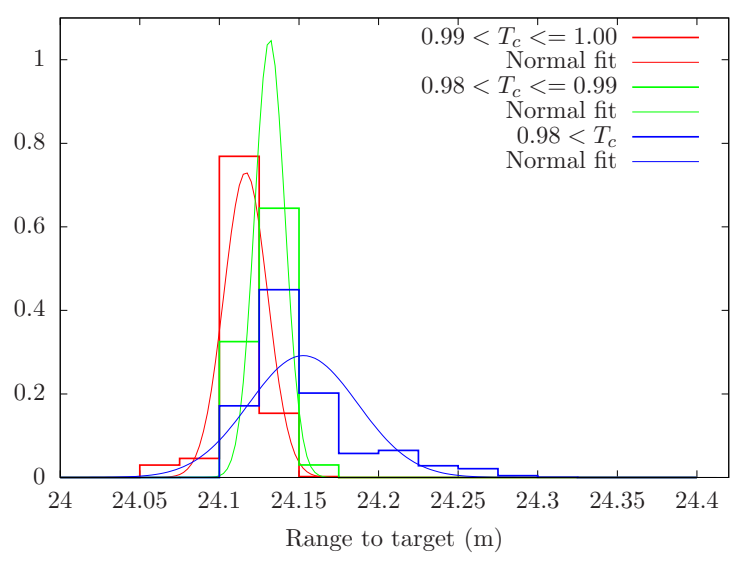

(a) SICK LMS (25m target)

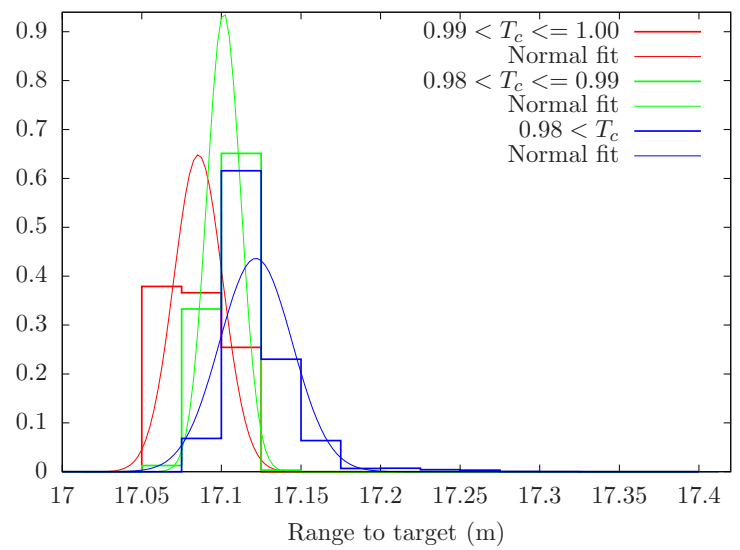

(c) SICK LMS (17m target)

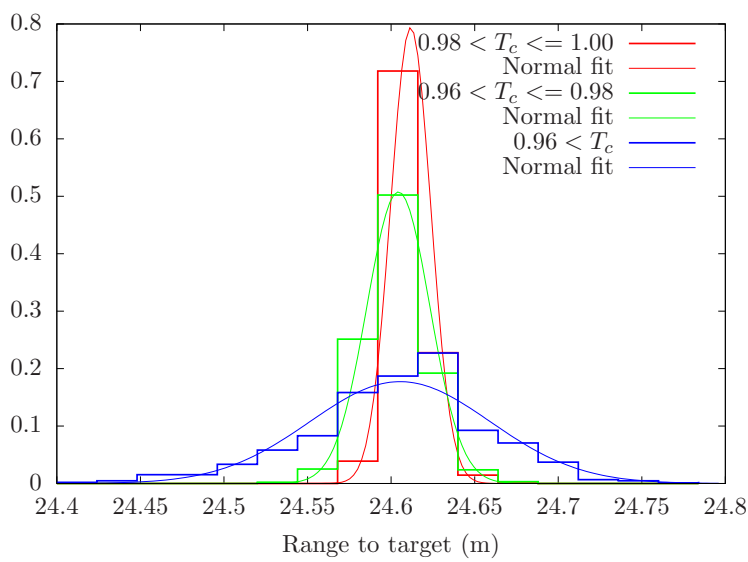

(b) Riegl (25m target)

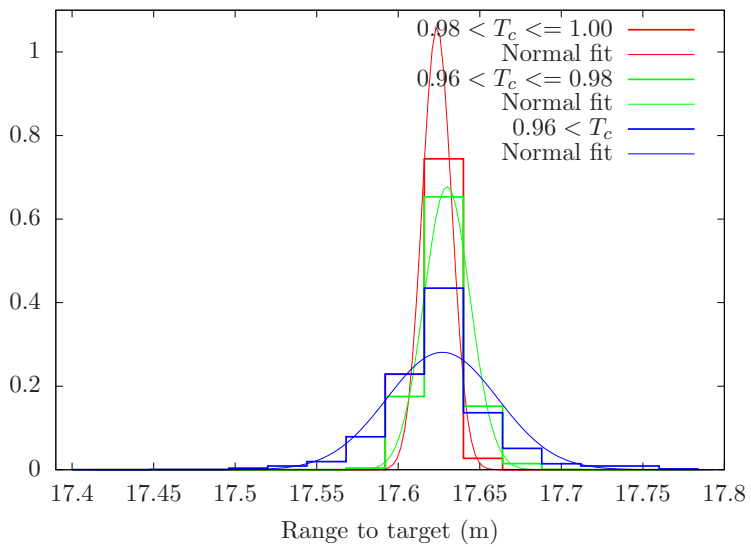

(d) Riegl (17m target)

Figure 8: Distribution of range returns for the laser sensors for various transmission coefficients. In $8 \mathrm{a}$ and $8 \mathrm{c}$ there is evidence of a range bias as the dust levels increase for the SICK. In $8 \mathrm{~b}$ and $8 \mathrm{~d}$, an increase in dust levels does not consistently introduce a bias, but rather a significant increase in the distribution. 


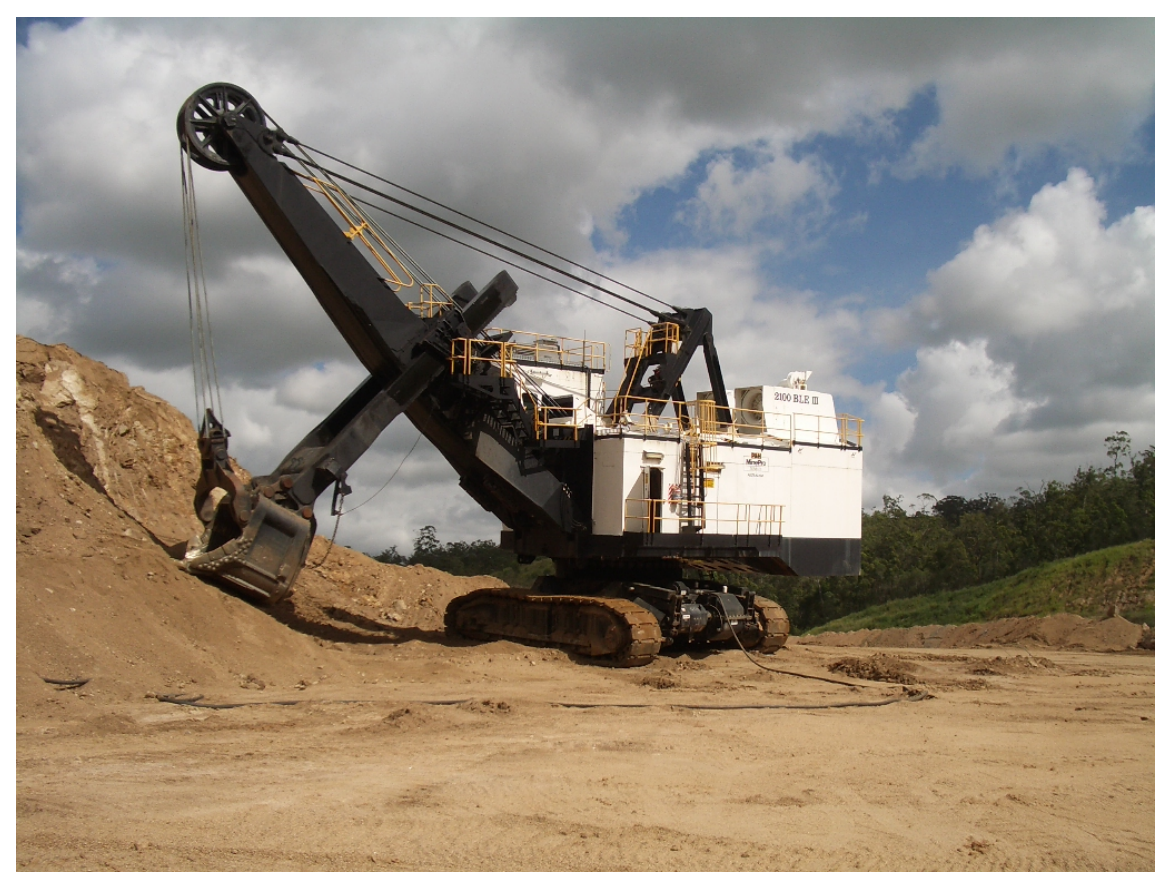

Figure 9: The P\&H 2100 BLE electric face shovel from which the field data presented in this paper was collected.

radar showed expected behaviour with lower point return densities and a higher uncertainty in the range and angular measurement than the lasers. This results in significant data noise with uncertainty in the ground plane's z-coordinate (vertical location) typically near $0.5 \mathrm{~m}$ and up to $1.25 \mathrm{~m}$ in some instances, although these extreme values are partly due to the 1st point return interpretation method employed in combination with the wide beam-width introducing a range bias dependant on the incidence angle. This uncertainty in measurement and low cloud density made identification of objects less than $2 \mathrm{~m}$ in size difficult in the radar data.

There is good correspondence between the filtered point clouds generated by the radar and those by the SICKs, with similar surfaces providing returns (generally those with a high angle of incidence to the beam) and both sensors were unable to see a region of standing water near the shovel due to the large angle of incidence. The radar occasionally showed spurious returns near items with a high-metallic content (e.g. the haul truck shown in Fig. 12) when the side-lobes of the radar signal intercepted the metal item. In these cases, the sensor returned a signal with an intensity above the free-space clutter filtering threshold as detected by the beam side-lobe, but with angular offset aligned with centre of the beam.

The significantly lower data acquisition rates and scan-rates for the radar presented the most limiting constraint for the terrain modelling application presented here. For this application, data is required of stationary haul trucks or other loading units positioned near the shovel to allow loading. Typically each loading cycle is 30-45 seconds long and involves the shovel digging, swinging to the load point above the truck, releasing the load and returning to the bank for the next dig. Several such cycles are required to fill the load unit (truck). 


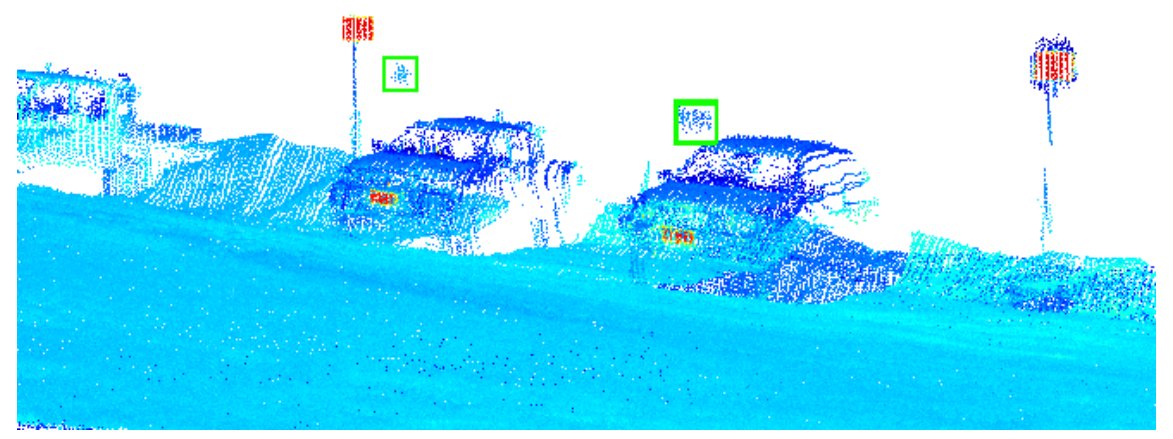

Figure 10: Point cloud data from the Riegl showing the effect of 'ghosts' in the data (highlighted in the green squares). Points are coloured by return intensity. The false points are due to the high intensity points immediately below from the vehicle number plates.

The data for the radar presented in Fig. 12 was collected over a period of approximately 11 minutes whilst the shovel was stationary, using the radar's pan axis actuation to give the 3D point cloud. This data shows a sparser point distribution than that collected by the laser in the Fig. 12, which was collected over approximately 17 seconds during a simulated loading cycle.

Fig. 13 summarises the associated distances between two point clouds from a subset of the data (truck) presented in Fig. 12. The SICK LMS point cloud was used as a base data set, and each radar point queried for its distance to the nearest point in the laser data. Assuming that the laser data is locally spatially correct, an assumption supported by visual inspection of the truck and its flat surfaces, the distribution in Fig. 13b of these nearest neighbour distances illustrates the error in the radar data. Specifically the presence of large $(2 \mathrm{~m})$ outliers and the poisson-like distribution of the errors is notable in both Fig. 13a and Fig. 13b. This highlights that although the radar operation is relatively unaffected by dust and rain its accuracy and data acquisition speed is currently insufficient for recognising objects without radar beacons, accurate (better than $0.5 \mathrm{~m}$ ) volume estimation or terrain modelling.

\section{$5 \quad$ Summary and Conclusion}

This paper has presented a repeatable, reliable and relatively inexpensive method for the comparison of the millimeter-wave radar and near infra-red laser scanning range finding technologies under varying environmental (dust, rain/mist) conditions. The metrics of range accuracy, target acquisition probability and signal intensity were compared to determine the threshold values at which the various sensing technologies failed under adverse environmental conditions. Further observations from testing in a field deployment were also presented.

It was found that in the environmental conditions tested indoors, the lasers showed accurate and reliable target detection when visual targets were no longer discernible by passive means, such as video imagery. There is a limit to the level of obscurity through which the lasers were able to provide accurate ranging information. This limit was similar for both the Riegl 


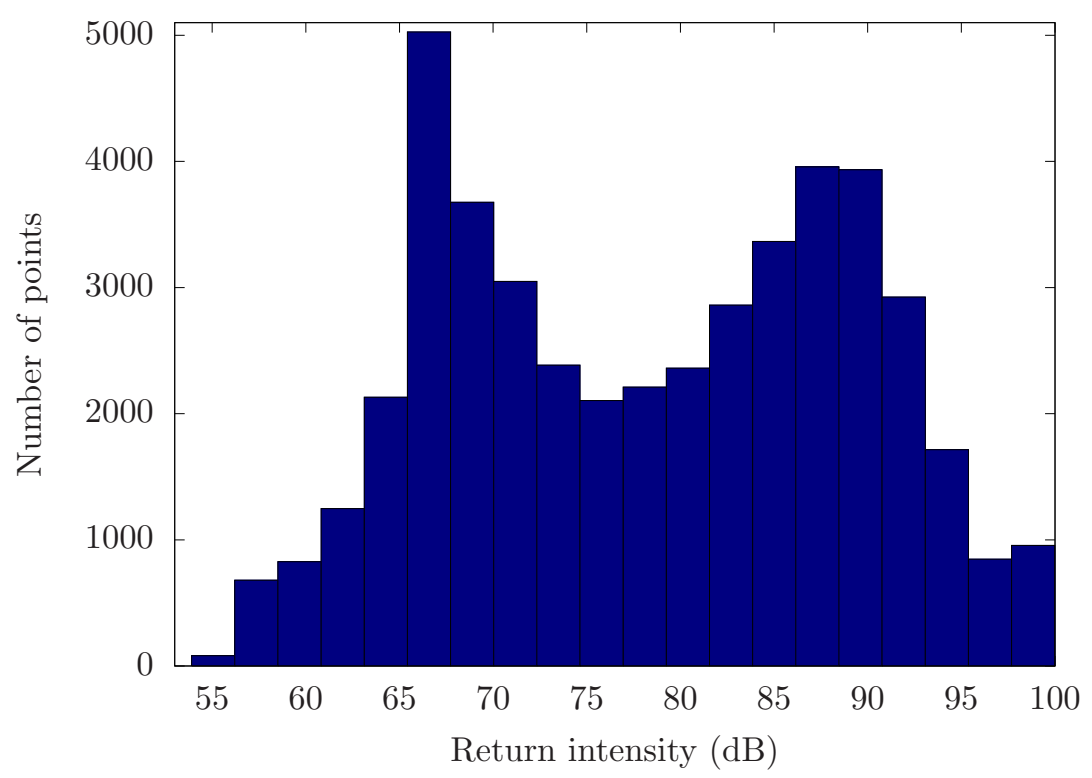

Figure 11: Distribution of radar point return intensities. The minima around $75 \mathrm{~dB}$ defined the initial threshold intensity used to remove free-space data clutter.

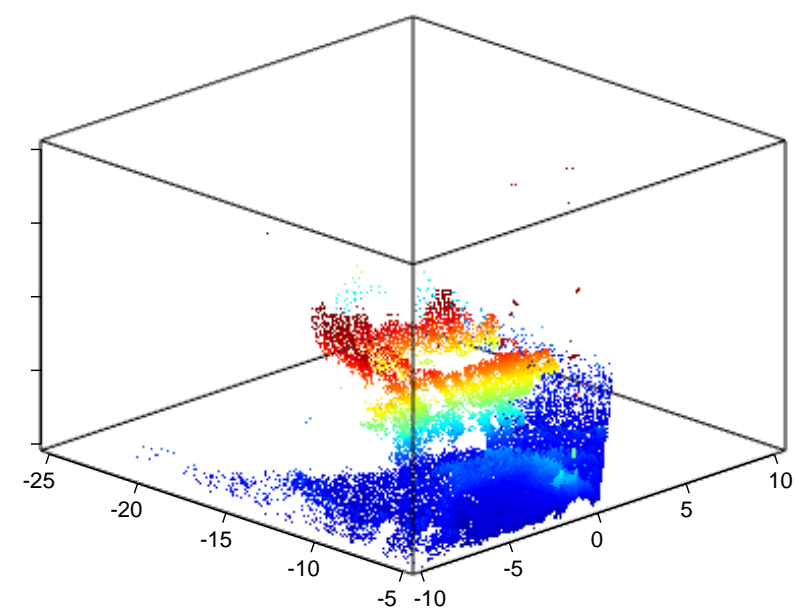

(a) Radar (680 seconds, 42,854 points)

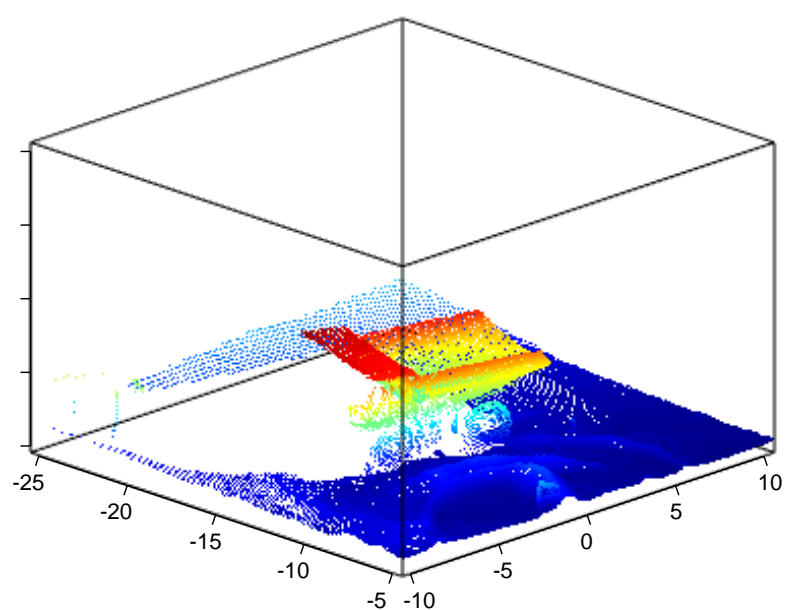

(b) SICK LMS (17 seconds, 51,666 points)

Figure 12: Point cloud data from the radar obtained while the shovel is stationary (12a) and the same scene as seen by a SICK laser during a shovel rotation (12b). The points are coloured by height to aid visual clarity. The laser scan represents 17 seconds of data acquisition (and 1/3 ${ }^{\text {rd }}$ data decimation for plotting: 51,666 points total displayed) versus the 680 seconds of data presented for the radar (and filtering at a 78dB threshold: 42,854 points total). 


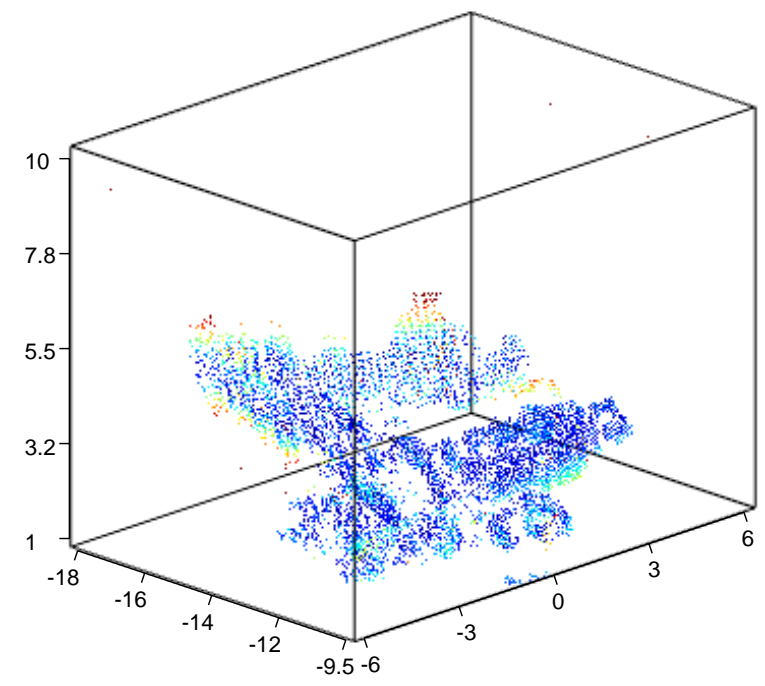

(a) Radar truck error

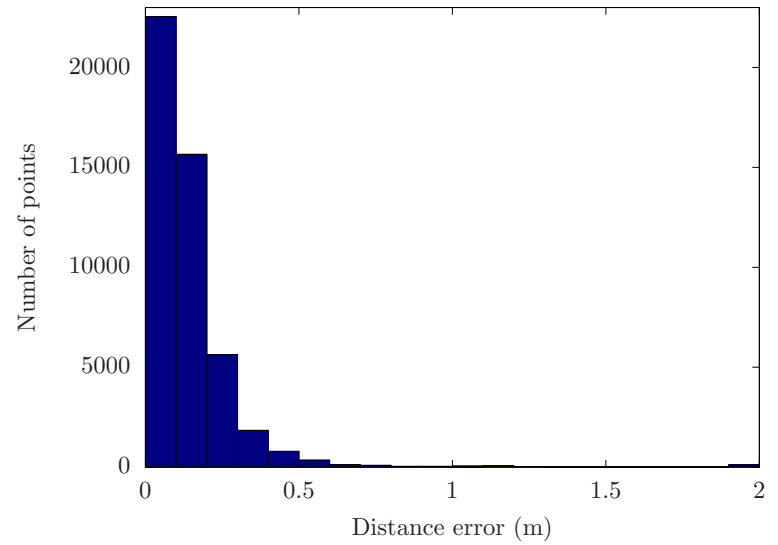

(b) Radar distance error distribution

Figure 13: Nearest neighbour associated distance error plots for the truck points presented in Fig. 12. The laser data is considered as a ground truth and each radar point coloured by its distance from the nearest laser point. (13a). The distribution of these error distances is presented in $13 \mathrm{~b}$ with $0.1 \mathrm{~m}$ interval spacing.

and SICK at a transmission coefficient of $92-93 \%$ per meter for bright targets closer than $25 \mathrm{~m}$.

For the testing conducted within the scope of this work, it appears that the false returns may be either so called phantom points (or mixed pixels [Adams and Kerstens, 1996, Ye and Borenstein, 2002]) formed by the combination of the returns generated by the rain, mist or dust and the target, or simply returns produced by the rain, mist or dust itself. These artifacts can be filtered out and there is some literature on common approaches (see, for example [Tuley et al., 2005, Tang et al., 2007]). Absent returns for a bright target occur only when the transmission coefficient is less than 92.5\%. In these poor conditions, the SICK LMS291-S05 tends to oscillate between false and absent returns. The Riegl provides a multiecho target discrimination mode, and during the rain tests there was a higher likelihood of the sensor returning a valid measurement when using the last echo return.

Both laser sensors provide a measure of the intensity of the return pulse: a simple integer gain measurement from 0 to 7 for the SICK LMS and 0 to 255 for the Riegl. The value of the return intensity correlated well to the transmission measurement for the Riegl, and under these controlled conditions could be used to detect when the sensor is likely to provide false readings. It is unlikely that such a method would be easily adapted to use in the field without discarding a significant portion of valid measurements from targets with low reflectance. The use of the return intensity as a means to determine the likelihood of a corrupt range measurement for the SICK LMS is not recommended as the observed intensities during the tests were low and showed minimal correlation to transmission measurements. The SICK 
LMS291-S05 is equipped with pollution sensing capability, however this only activated when there was a significant build up of dust on the surface lens of the sensor and did not appear to be a robust method of discriminating between false/absent and real returns during testing.

The radar return was relatively unaffected by the generated conditions, showing robust behaviour. However, as a sensing technology radar provides lower range and angular precision than the laser systems. This is mainly due to its wider beam width and longer wavelength. The radar tested here exhibits other technology specific behaviours which make generation of digital terrain maps difficult and limits application of the sensor to those operations without a need for high fidelity or high sample rates.

The radar showed reliable performance and accuracy irrespective of the intermediary obscurant density for the point reflector targets used in these tests. Sensor return intensity showed negligible attenuation in the dust trials, but dropped approximately $3 \mathrm{~dB}$ under the highest rain rates.

The results from this study indicate the following:

1. Radar generates point-cloud type information that is robust to adverse environmental conditions including heavy mist, rain and dense dust clouds. The measurements are of low precision and in our application, spatially sparse, but sufficient for the provision of structure, and large $(\approx 2 \mathrm{~m})$ object segmentation.

2. Laser provides a more easily interpreted measurement ideally suited to the creation of digital models of the surrounding terrain with high precision and accuracy. The measurements are susceptible to corruption in environments with high mist and dust loading. This data degradation is not easily identified from the laser measurement alone.

3. There is scope to combine the output from the sensors to create a more robust representation of the surrounding terrain utilising the strengths of both sensors to compensate for the individual sensors' weaknesses. It is apparent that neither sensor alone is sufficient for the expected highly adverse environment of the mining operating space.

It is intended to develop and evaluate appropriate fusion methods and spatial representations for these sensors to address this last point. Some guiding heuristics to base this work on are:

- using radar returns to provide a 'rough-draft' of the surrounds in which significant obstacles are clearly identifiable and that is robust to adverse weather and dust;

- using laser information to provide the detail of the surrounds, and other information required for tasks such as volume estimation and classification of objects and obstacles;

- using radar returns to determine when information provided by the laser sensors have been degraded by adverse weather and dust.

The authors note that the conducted tests were specific to the project within which they were conducted (ACARP project C16031). The effects of varying the composition of the 
dust to include materials which have the potential for a high radar cross section (e.g. iron ore dust), large rain drops (such as may be encountered during tropical storms), ice (hail or snow) were not specifically considered. These conditions have the potential to further affect the sensor returns from both sensing technologies and may lead to additional effects being observed.

All our indoor experiments considered situations where the sensors were within the obscuring media 'cloud'. A highly reflective cloud that is separated from the sensor by intervening clear space will inevitably produce detection of an inexistant object (the edge of the cloud) if the laser sensor is used alone (versus some fusion method against the radar). This case was not explicitly considered. Similarly the absence of an obstacle due to high absorption properties of the cloud may also cause system failure if the laser sensor is used alone, however we have shown that the likelihood of this failure can be evaluated through simple experiments and measurement of the transmission in environments that reflect the deployment.

Adverse environmental conditions as severe as those generated in the controlled testing are yet to be observed in the field and indicate an area of required further work. It would be desirable to observe and assess the sensors' performance over an extended period in a production environment. Furthermore, it is suggested that any such testing aim to incorporate as varied operating conditions as possible, including operation in significantly different ore-types, for example coal and iron.

\section{Acknowledgment}

The authors would like to thank the Australian Coal Association Research Program (ACARP) for funding project C16031 which provided for the work presented in this paper. We would also like to thank the joint partners in this research project: the Cooperative Research Centre for Mining (CRCMining) and the Australian Centre for Field Robotics (ACFR). In particular, we would like to thank (from CSIRO) Mike Bosse, for laser sensor calibration and technical advice on the data acquisition and processing, Steve Nuske for providing the camera response curve (from ACFR) Javier Martinez and Graham Brooker for provision and calibration of the radar, providing the results of the radar performance under controlled conditions and review feedback on a report from which this paper is based, and (from CRCMining) Ross McAree, Anthony Reid, Kevin Austin, Rebecca Hill and Andrew Denman for managing the ACARP project, providing access to and operating the test facilities including the research shovel.

\section{References}

Adams, M. and Kerstens, A. (1996). The interpretation of phase and intensity data from AMCW light detection sensors for reliable mapping. Int J. of Robotics Research, $15(5): 441-458$.

Arras, K. O. (2003). Feature-Based Mobile Robot Navigation in Known and Unknown Environments. PhD thesis, Swiss Federal Institute of Technology Lausanne (EPFL).

Arroyo-Suarez, E. N., Riley, J. L., Glang, G. F., and Mabey, D. L. (2005). Evaluating a 
global differential GPS system for hydrographic surveying. In OCEANS, Proceedings of MTS/IEEE, volume 3, pages 2557-2563, Washington DC.

Baker, C., Morris, A., Ferguson, D., Thayer, S., Whittaker, C., Omohundro, Z., Reverte, C., Whittaker, W., Hhnel, D., and Thrun, S. (2004). A campaign in autonomous mine mapping. In Proceedings of the 2004 IEEE Int. Conf. on Robotics and Automation.

Brooker, G., Hennesy, R., Lobsey, C., Bishop, M., and Widzyk-Capehart, E. (2007). Seeing through dust and water vapor: Millimeter wave radar sensors for mining applications. Journal of Field Robotics, 24(7):527-557.

Brooker, G., Hennesy, R., Scheding, S., and Bishop, M. (2005). Development and application of millimeter wave radar sensors for underground mining. IEEE Sensors Journal, 5(6):1270-1280.

Corke, P., Roberts, J., and Winstanley, G. (2000). Experiments and experiences in developing a large robot mining system. In Experimental Robotics VI, volume 250/2000, pages 183192, Berlin / Heidelberg. Springer.

Diosi, A. and Kleeman, L. (2003). Uncertainty of line segments extracted from static sick pls laser scans. In Australasian Conf. on Robotics and Automation, Brisbane, Australia.

Duff, E. (2006). Accurate guidance and measurement for excavators using a laser scanner. Technical Report C14043, ACARP.

Duff, E., Usher, K., and Ridley, P. (2006). Swing loader traffic control. Technical Report C13041, ACARP.

Foessel, A. (2000). Radar sensor model for three-dimensional map building. In Proc. SPIE, Mobile Robots XV and Telemanipulator and Telepresence Technologies VII, volume 4195. SPIE.

Gillett, D. (1979). Environmental factors affecting dust emission by wind erosion. Wiley.

Hall, D. and Keays, J. (1993). Dozer trak system phase 2. Technical Report C3045/C3056, ACARP.

Huber, D. and Vandapel, N. (2006). Field and Service Robotics: Recent Advances in Research and Applications, volume 24 of Springer Tracts in Advanced Robotics, chapter Automatic $3 \mathrm{D}$ underground mine mapping. Springer.

Macfarlane, D. and Robertson, D. (2004). AVTIS - a dual-mode imaging millimetre wave radar/radiometer for volcanological surveying. Geoscience and Remote Sensing Symposium, 2004. IGARSS '04. Proceedings. 2004 IEEE Int., 5:3299-3302 vol.5.

Martinez, J. (2007). Rain simulator design. Technical Report Internal, ACFR.

Miller, I., Campbell, M., Hutteniocher, D., Kilne, F.-R., Nathan, A., Lupashin, S., Catlin, J., Schimpf, B., Moran, B., Zych, N., Garcia, E., Kurdziel, M., and Fujishima, H. (2008). Team Cornell's Skynet: Robust perception and planning in an urban environment. Journal of Field Robotics, 25(8):493-527.

Noon, D., Reeves, B., Sticley, G., Holmstrom, M., Smith, G., and Longstaff, D. (2002). Development of slope stability radar. Technical Report C10036, ACARP.

Nüchter, A., Surmann, H., Lingemann, K., and Hertzberg, J. (2004). 6D slam with an application to autonomous mine mapping. In Proceedings of the 2004 IEEE Int. Conf. on Robotics and Automation. 
Pinnick, R., Fernandez, G., and Hinds, B. (1983). Explosion dust particle size measurements. Applied Optics.

Reeves, B., Stickley, G., Noon, D., and Longstaff, I. (2000). Developments in monitoring mine slope stability using radar interferometry. Geoscience and Remote Sensing Symposium, 2000. Proceedings. IGARSS 2000. IEEE 2000 Int., 5:2325-2327 vol.5.

RIEGL Gmbh (2005). LMSQ120 technical documentation and users instructions. A-3580 Horn, Austria.

Roberts, J., Winstanley, G., and Corke, P. (2003). 3d imaging for a very large excavator. Int. Journal of Robotics Research, 22(7/8):467-478.

Scheding, S., Brooker, G., Hennesy, R., Bishop, M., and Maclean, A. (2002). Terrain imaging and perception using millimeter wave radar. In Proc. 2002 Australasian Conf. on Robotics and Automation, pages 60-65, Auckland.

Shaffer, G., Stentz, A., Whittaker, W., and Fitzpatrick, K. (1992). Position estimator for underground mine equipment. IEEE Transactions on Industry Applications.

SICK AG (2000). LMS291 technical description. Auto Ident Germany.

Singh, S. (1997). The state of the art in automation of earthmoving. ASCE Journal of Aerospace Engineering, 10(1).

Stentz, A. T., Bares, J., Singh, S., and Rowe, P. (1999). A robotic excavator for autonomous truck loading. Autonomous Robots, 7(1):175-186.

Tang, P., Huber, D., and Akinci, B. (2007). A comparative analysis of depth-discontinuity and mixed-pixel detection algorithms. In Sixth Intl. Conf. on 3-D Digital Imaging and Modelling (3DIM 2007), Montral, Canada.

Tuley, J., Vandapel, N., and Hebert, M. (2005). Analysis and removal of artifacts in 3-d ladar data. In Proceedings of the 2005 IEEE Int. Conf. on Robotics and Automation, Barcelona, Spain.

Widzyk-Caperhart, E., Brooker, G., Hennesy, R., Lobsey, C., and Scheding, S. (2006). Application of millimeter wave radar sensor to environment mapping in surface mining. 9th Int. Conf. Control, Automation, Robotics and Vision, pages 1-6.

Yamauchi, B. (2007). Daredevil: Ultra-wideband radar sensing for small UGVs. In Proceedings of SPIE, volume 6561, Orlando, Florida, USA.

Ye, C. and Borenstein, J. (2002). Characterization of a 2-D laser scanner for mobile obstacle negotiation. In Proceedings of the 2002 IEEE Int. Conf. on Robotics and Automation, pages 2512-2518, Washington DC. 